\title{
ULTRASONIC GUIDED WAVE TOMOGRAPHY FOR THE INSPECTION OF THE FUEL TANKS FLOOR
}

\author{
L. Mažeika, R. Kažys, R. Raišutis, R.Šliteris \\ Ultrasound Institute, Kaunas University of Technology, Lithuania
}

\begin{abstract}
The novel ultrasonic NDT technique based on application of long range guided waves is proposed for detection of defects in a storage tank floor. The technique needs access only to the outer edge of the tank floor and do not require emptying and cleaning inside of the tank. The technique has been developed on the basis of the Lamb wave transmission tomography. The propagation of the guided waves in the tank floor has been carried out using simulation and experimental verification. The results obtained on the reduced size tank and results of in situ experiments are presented
\end{abstract}

Key words: long range ultrasonic NDT, tank inspection, corrosion type defects, ultrasonic tomography, guided waves.

Contact author: Liudas Mažeika, Phone: +370 37 351162, Fax: +370 37 451489, e-mail:

liudas.mazeika@ktu.lt

Ultrasound Institute of Kaunas University of Technology, Studentu st. 50, Kaunas LT-51368, Lithuania

\section{Introduction}

In petrochemical industry corroded areas in a fuel tank floor is an object of ultrasonic non-destructive testing (NDT). Various NDT methods for inspection of tanks, such as penetrant testing, magnetic particle, radiographic testing, eddy current, thermography, acoustic emission and also ultrasonic techniques are used [1,2]. The inspection of storage tanks is a time consuming and expensive procedure, mainly due to necessity to empty and clean the tank before the inspection using conventional NDT methods. Therefore, the objective of this study was to develop an ultrasonic technique, suitable for tanks inspection without emptying and cleaning the tank. The most promising technique which enables inspection at a relatively long range and can be used for inspection of a tank floor from an outside perimeter is based on exploitation long range ultrasonic guided waves. The low frequency guided waves can propagate long distances in planar and tubular structures and already are used for inspection of pipes [1,2]. The main differences of the tank floor inspection case is complexity of the object containing multiple lap joint welds along the large diameter of the tank (up to $100 \mathrm{~m}$ ) and necessity to reconstruct 2D distribution of defects from the information obtained at the edges of a tank floor or external vertical walls.

The main scope of the investigation was application of the ultrasonic transmission tomography for localization of non-uniformities inside tank floor, taking into account ultrasonic signal losses due to loading of a tank floor by the liquid in a tank and a basement (sand) on which the floor is laid down.

\section{Application of the guided waves for NDT of the petroleum tank floor}

The tank floor consists of the set of steel plates having thickness 6-8 $\mathrm{mm}$ joined together using lap welds and may have the diameter up to $100 \mathrm{~m}$ (Fig. 1(a, b)). The guided waves propagate in planar structures such as plates or sheets and may possess unlimited number of modes. Mainly the symmetric $\mathrm{S}_{0}$ or asymmetric $\mathrm{A}_{0}$ modes are used for inspection. The transmission, reflection and mode conversion of these waves takes place on each non-uniformity of the floor, including welds and defects. Each of these phenomena causes energy losses of propagating waves. On the other hand, that enables detection of defects using reflection or transmission techniques [1-3]. 

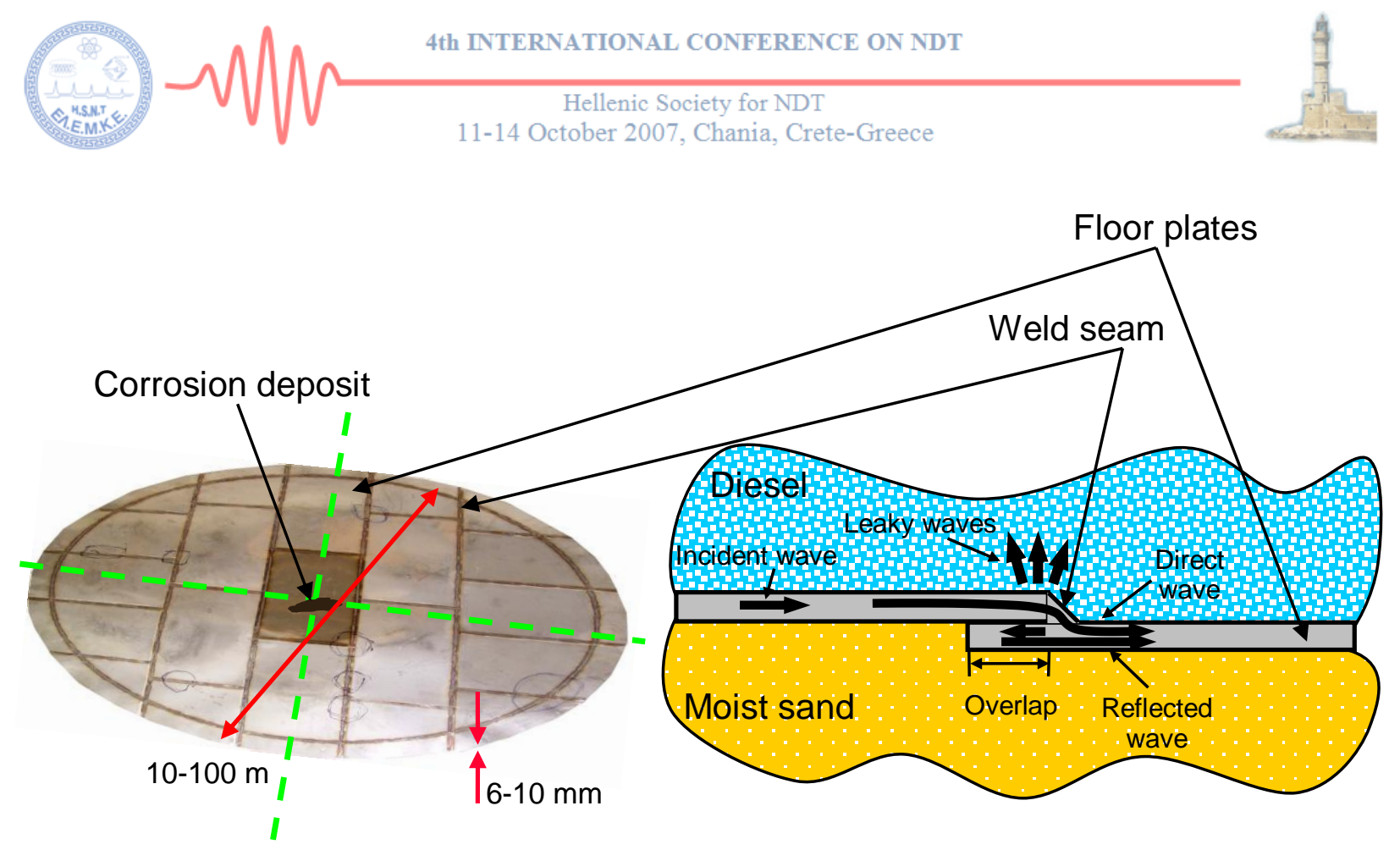

(a)

(b)

Fig.1. The typical geometry of a tank floor (a) and the model of Lamb wave propagation through a lap joint weld (b)

In order to develop an efficient testing technique regularities of Lamb wave propagation through tank floor plates and welds loaded by the sand from a bottom side and by the fuel (diesel) on the top side have been investigated. The theoretical investigation was carried out to estimate the losses of the Lamb waves propagating in a tank floor using the finite difference method. The obtained regularities have been verified experimentally. It was determined also that the most critical parameters determining losses of the $\mathrm{S}_{0}$ mode are the boundary conditions between the steel plate and a supporting medium, for example, wet sand. The simulation and experiments have shown that the contact between the mentioned media is a slip, that is, the shear component of the waves is not transmitted into another medium. It was found that the losses of the $\mathrm{S}_{0}$ mode guided wave in the tank floor loaded by diesel from one side and wet sand from another side at the 50 $\mathrm{kHz}$ are approximately $0.5 \mathrm{~dB} / \mathrm{m}$. Such attenuation corresponds to "slip" boundary conditions between these materials [4]. In the case of a medium size tank with the diameter $30 \mathrm{~m}$ this leads to $15 \mathrm{~dB}$ attenuation. In the case of a "solid" contact, like gluing, the losses of the $S_{0}$ mode may increase up to $10 \mathrm{~dB} / \mathrm{m}$. The losses of the $\mathrm{A}_{0}$ mode wave are more than $1 \mathrm{~dB} / \mathrm{m}$ under the same conditions, so, taking into account possible diameters of the tank this mode is not suitable for a long range inspection.

Numerical simulation and experimental investigations also have shown that the transmission losses of the $S_{0}$ mode wave propagation through welded lap joint are additive in the direction perpendicular to the weld seams [4-6]. The mentioned effect can by be explained by interference of two signals: the signal transmitted through the weld and the signal reflected from the left end of the bottom plate. It was also found that the losses are frequency dependent due to interference of the transmitted and reflected signals (Fig. 1 b). There can be tens of welds across diameter of the tank floor, so the total losses may exceed 100 dB.

\section{Regularities of the guided waves propagation through the welded tank floor}

The previous investigations have demonstrated that the $S_{0}$ mode of Lamb waves, generated at the $50 \mathrm{kHz}$ frequency, enables through transmission measurements of the floor of small and medium size storage tanks with diameters $5-30 \mathrm{~m}$. The propagation of the $S_{0}$ mode of Lamb wave in a steel plate and its 
interaction with non-uniformities, such as artificial and corrosion defects were investigated. It was shown that the most promising approach for detection of corroded areas in a tank floor should be based on the through transmission tomography of Lamb waves. The inspection technique based on such an approach have been developed and tested experimentally [4-6].

The main task of the second stage of investigation was to determine accuracy limits and to optimize necessary parameters of the measurement, minimizing the number of measurements which should be performed on a tank in situ. To achieve this task the scaled physical model of a real tank was developed. The measurements were performed at properly scaled higher frequencies. The adequacy of measurement conditions have been verified using modelling and experimental investigations. The experiments carried out on the scaled model and the developed signal and data processing procedures enable to visualise dynamics of ultrasonic fields over the plate and determine the position of various defects. The signals acquired during experiments have demonstrated a good agreement with the numerical simulation. It was shown that the transmission tomography based on the measurement of the Lamb waves, propagating in a tank floor, enables to obtain a spatial distribution of attenuation of Lamb waves in a tank floor, what corresponds to the corroded areas of the floor. The accuracy and the possibilities of the developed technique are demonstrated using the results of the experiments on the scaled model of the tank and the tank in situ [4-6].

For experimental investigation the scaled down size tank mock-up (scaling factor 8:1) placed on wet sand and filled with water was selected (Fig.2a). The internal diameter of the tank was $1 \mathrm{~m}$, the height 1.1 $\mathrm{m}$, the thickness of the floor plate was $1 \mathrm{~mm}$. The experimental set-up used in the investigation and the plates arrangement of the welded floor is presented in Fig. $2 \mathrm{~b}$.

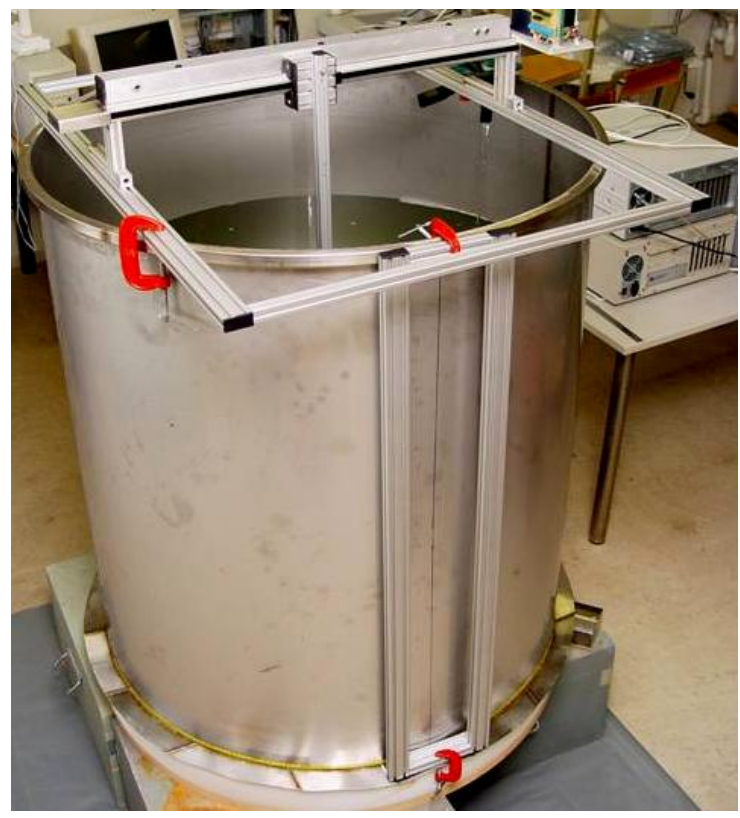

a

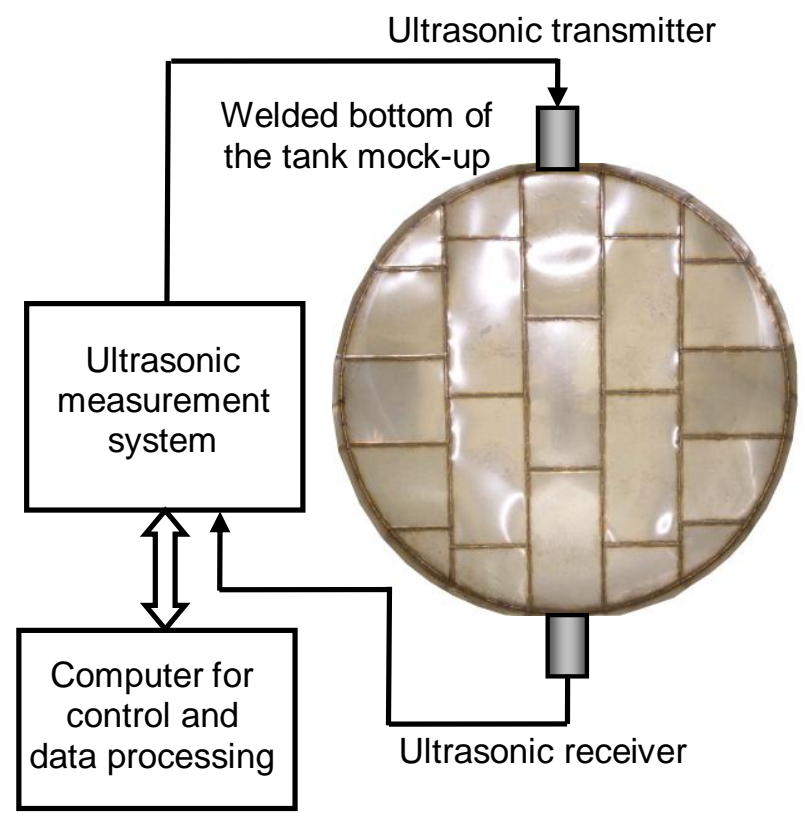

b

Fig.2. Photo of the 1:8 scaled down size tank mock-up (a) and the measurement set-up (b) used for investigation

The transmitting ultrasonic transducer was mounted on the edge of the plate. Data acquisition was performed by the measurement system developed in the Ultrasound Institute of Kaunas University of Technology. The excitation pulse frequency was $400 \mathrm{kHz}$, the number of the rectangular burst cycles was 3. The excitation voltage of the transmitter was $200 \mathrm{~V}$ and the gain of the receiver was $50 \mathrm{~dB}$. The receiving transducer was mounted on the edge of the tank floor (flange). The signals propagating through 
the tank floor were recorded. The fundamental $\mathrm{A}_{0}, \mathrm{~S}_{0}$ and the multiple mode conversion signals have been observed. The mode conversion $\left(\mathrm{A}_{0}\right.$ mode to $\mathrm{S}_{0}$ and opposite) take place at each weld of the bottom plate and on the weld between the floor and wall plates.

The measured leakage losses of the $S_{0}$ mode in the floor of the scaled down size tank mock-up (the diameter $1 \mathrm{~m}$ and the thickness $1 \mathrm{~mm}$ of which correspond to the diameter $8 \mathrm{~m}$ and the thickness $8 \mathrm{~mm}$ of the real tank), which was resting on moist sand and loaded by water from the top side are: $(5.5 \pm 0.6) \mathrm{dB}$. The calculated losses using the global matrix method [4] are $4.7 \mathrm{~dB}$ (at $50 \mathrm{kHz}$, the thickness of the floor plate $8 \mathrm{~mm}$ and the diameter $8 \mathrm{~m}$ ). The calculated losses using the finite difference method [4] are $6.1 \mathrm{~dB}$ (at $50 \mathrm{kHz}$, the thickness of the floor plate $8 \mathrm{~mm}$ and the diameter $8 \mathrm{~m}$ ). The experimental verification of the $S_{0}$ mode attenuation, caused by propagation through a few welds, was performed using the scaled down size physical model. A good agreement is obtained between the simulated and the measured results. The estimated attenuation of the $S_{0}$ mode transmitted through a weld in the direction perpendicular to the weld seams was (7.95 \pm 0.76$) \mathrm{dB}$ and through the two welds was (15.9 \pm 1.5$) \mathrm{dB}$ (Fig.3 a). That corresponds very well to the results $(7.8 \mathrm{~dB})$ obtained by modeling using the finite difference method, also proves the assumption about additive transmission losses [4].

In order to analyze leaky waves, which were radiated into liquid by non-uniformities inside the tank floor, the immersion type receiving transducer was scanned over the segment of the tank floor welded plates. The mentioned segment was also affected by a natural corrosion. The transformations of the $\mathrm{S}_{0}$ mode into $\mathrm{A}_{0}$ mode, propagating almost uniformly in all directions, have been observed at each weld and a corrosion type defect. Therefore, it was assumed that the non-uniformity inside a tank floor acts as a virtual transmitter of the $A_{0}$ mode wave $[4,6]$.

Due to required big computational resources it is impossible to simulate propagation of ultrasonic waves through the whole bottom of the petroleum tank. Therefore the computational task was decomposed into separate subtasks, like analysis of the signal propagation through the loaded steel plate without welds and the signal propagation through weld seam of two welded plates [4].

During numerical investigation of the fundamental symmetric $S_{0}$ mode, propagating through welded plates, it was found that the interference of multiple reflected waves in each zone of weld seam occur. It was estimated, that the frequency dependent attenuation of the signal transmitted through a welded lap joint is a function of the plates overlap width and the attenuation in the lap joints is additive in the direction perpendicular to the weld seams. The attenuation caused by a single weld is minimal at the frequencies where multiple reflections are adding up with the same phase and the attenuation is bigger where reflections are adding up with the opposite phase [5].

The waveform of the signal transmitted through the whole bottom structure may be predicted [8] from the apriori known incidence (reference) signal $u_{\text {ref }}(t)$ if the complex transfer function $H_{\text {plate }}(j \omega, x)$ of the loaded plate (by diesel and moist sand) and the complex transfer function $H_{\text {weld }}(j \omega, m)$ of the weld under investigation are known:

$$
u_{\text {trans }}(t)=\operatorname{IFT} \bigcup_{\text {ref }}(j \omega) \cdot H_{\text {plate }}(j \omega, x) \cdot H_{\text {weld }}(j \omega, m),
$$

where $H_{\text {plate }}(j \omega, x)$ is the complex transfer function in a through transmission mode of the loaded steel plate of an arbitrary length $x, H_{\text {weld }}(j \omega, m)$ is the complex transfer function in the through transmission mode of a single weld with the wave propagation path in the welded plates (loaded by diesel and moist sand) equal to $1 \mathrm{~m}, j=\sqrt{-1}, U_{r e f}(j \omega)$ is the Fourier transform of the reference signal $u_{r e f}(t)$ which is picked up by the ultrasonic transducer placed in front of the welded zone, IFT denotes the inverse Fourier transform, $m$ is a number of the welds in the tank floor arrangement. The model of the petroleum tank bottom, which is welded by lap joints is presented in Fig.1.

The complex transfer function of the bottom structure (which length is $x$ ) without zones of the welded lap joints is given by:

$$
H_{\text {plate }}(j \omega, x)=\mathrm{e}^{-k \cdot x \cdot a-j \cdot \frac{x \cdot \omega}{v_{p}(\omega)}}
$$


where $\alpha(\omega)$ is the attenuation coefficient due to the leakage to surrounding media (diesel and sand) in $\mathrm{dB}$ calculated by numerical simulation technique [4,5], $k$ is a conversion coefficient from $\mathrm{dB}$ to $\mathrm{Np}(k=0.115)$, $x$ is propagation distance $(\mathrm{m})$ of the ultrasonic wave in the region of the floor plate without welds, $v_{p}(\omega)$ is a phase velocity $(\mathrm{m} / \mathrm{s})$ calculated by the numerical simulation technique [4-6].

The approximation of the complex transfer function magnitude of the single weld by linearly increasing harmonic oscillations, in order to achieve narrow -band filtering effect of the transmitted signal, is given by:

$$
H_{\text {weld }}(j \omega, m)=\mathrm{e}^{-k \cdot m \cdot\left(\alpha_{0} \cdot \frac{\omega}{\omega_{0}}+A-A \cdot\left|\sin \left(\omega t-t_{0}\right)\right|\right)-j \cdot m \cdot \Delta \Theta},
$$

where $\alpha_{0}$ is the attenuation coefficient in $\mathrm{dB}$ at the frequency $\omega_{0}$, in the presented case $\alpha_{0}=(3.91 \pm 0.4) \mathrm{dB} / \mathrm{m}$ at $f_{0}=66.3 \mathrm{kHz}, m$ is a number of the welds, $\Delta \Theta$ is difference between the phase spectrum of the signal picked up before the weld and the phase spectrum of the signal transmitted through a single weld, $A$ is the amplitude of the harmonic oscillations generated by a harmonic function in order to mimic the attenuation function of the weld, $t_{0}$ is the initial time shift of the harmonic function. The measured and predicted waveforms of $S_{0}$ mode wave in front of and behind the welded lap joint are presented in Fig.3 a and $b$. These results are relevant in the case of the analysis of the broadband ultrasonic pulse propagation through the welded plates of a petroleum tank floor.

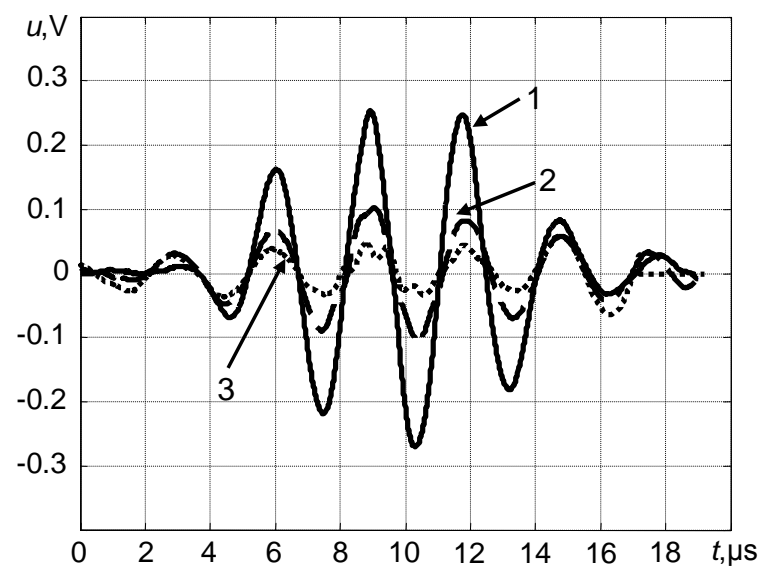

(a)

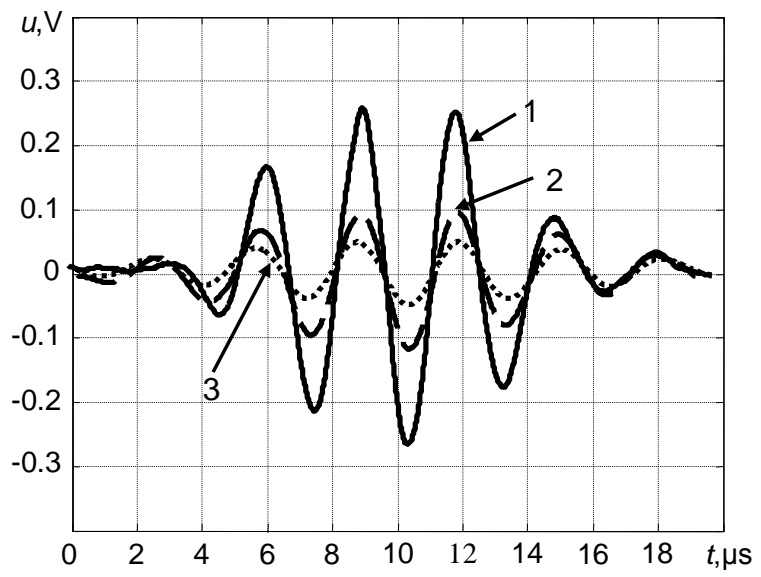

(b)

Fig.3. The measured (a) and simulated (b) waveforms of $\mathrm{S}_{0}$ mode wave in front of and behind the welded lap joint: 1 - in front of the $1^{\text {st }}$ weld, 2 - behind the $1^{\text {st }}$ weld, 3 - behind the $2^{\text {nd }}$ weld

\section{Spatial localization of the non-uniformities by transmission tomography}

One of the most efficient methods which enables to reconstruct the spatial distribution of some physical parameter is tomography. The basic principle of it is based on multiple measurements across the object at different angles using fan type or linear beams and in such a way obtaining multiple so called projections. In the second step the back-projection using special algorithms is performed in such a way obtaining the distribution of the investigated parameter in the cross-section of the object. There are many versions of the ultrasonic tomography, but the transmission tomography was selected as the most promising one [2,7]. In this case the physical parameter, the spatial distribution of which should be reconstructed, is attenuation of the $S_{0}$ mode Lamb wave.

In order to estimate the performance of the developed reconstruction algorithm, the experimental measurements have been carried out in situ on a small $8 \mathrm{~m}$ diameter tank (Fig.4 a), with the step $25 \mathrm{~cm}$ along the circumference of the tank floor. The total number of measurement points was 441 . In the 
measurements $50 \mathrm{kHz}$ ultrasonic transducers were used. The transducers were attached to the edge of the tank floor using liquid coupling and magnetic fixing. The tank floor edge was prepared in advance by grinding. For the signal excitation and reception the ultrasonic imaging system developed by the Ultrasound institute of Kaunas University of Technology was used (Fig.4 a). The transmitters were excited using the three periods burst.

In such a way the number of projections was acquired. The projection in the ultrasonic transmission tomography is assumed to be the distribution of the attenuation versus transmission direction or angle (Fig.4 b) for one transmitter position. In our case the transmitter was attached to the edge of the tank floor on one side and receivers were placed at different points on the opposite side of the tank floor covering approximately $180^{\circ}$ sector. At each position from the acquired signals the relative attenuation (losses) are measured. The other projections are acquired in a similar way, only the transmitter is attached to the edge of the floor at different positions with some step from the previous one and the measurements on the opposite side of the tank in a corresponding sector are performed. In general, the tomography requires as much as possible of different projections, because the accuracy and resolution of the reconstructed images depend on the number of projections. On the other hand, the accuracy depends on the wavelength of the exploited Lamb wave. So, the optimal number of projections should be determined for each particular case. During investigations 9 tomographic projections were acquired (Fig. 4 b). To increase reliability and reduce a noise at each point 11 measurements were performed.

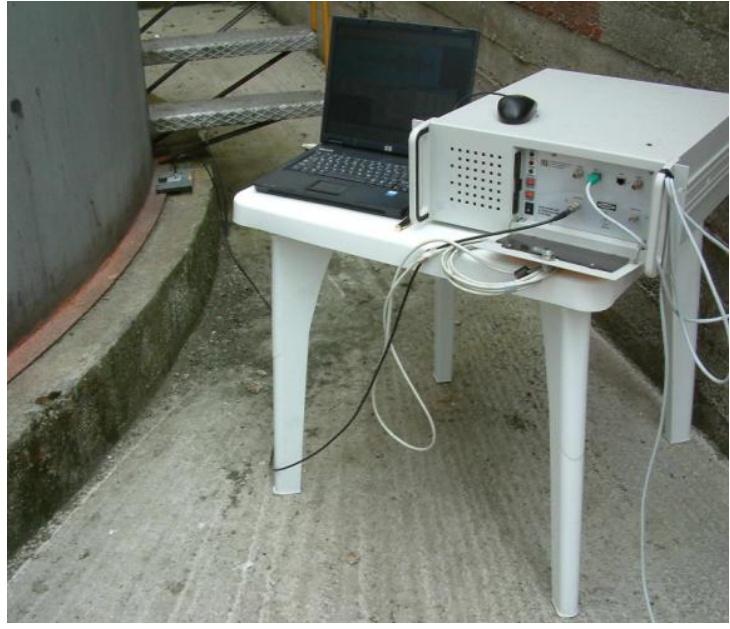

(a)

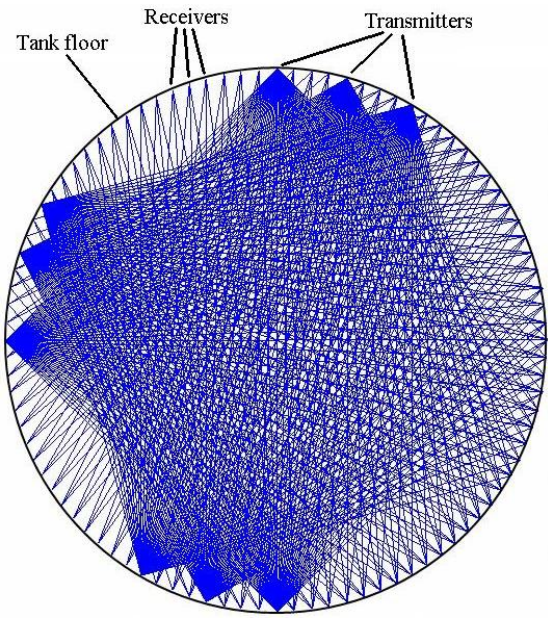

(b)

Fig.4. Set-up for application of the transmission tomography of a tank floor: a - photo of the real tank segment, which is necessary to inspect, b - the transmitter and receiver positions during in situ experiments

After acquisition the signals possessing the amplitude 10 times smaller then the amplitude of the maximal signal were excluded from the analysis and the average signal was calculated. In the next step only the front part of the signal, which corresponds to the propagating $S_{0}$ mode of the Lamb wave was selected. This mode propagates with a higher propagation velocity and should pass the tank floor first. That enables to select the $S_{0}$ mode in the time domain. The amplitude variations can be caused not only by some non-uniformities in the tank floor, but also by the directivity pattern of the transmitter. To exclude these phenomena from the analyzed data the average distribution was calculated and the approximate directivity pattern was obtained. This function will be used as the projection for tomographic reconstruction of distribution of the attenuation in the tank floor (Fig.5 a). In this figure $a_{\mathrm{xy}}$ is the angle of the point $P(x, y)$ under analysis with respect to the projection, $a_{k 1}$ and $a_{k 2}$ are the angles of the closest point of the projection, $k_{1}$ and $k_{2}$ are the numbers of the closest measured points, $A_{n}$ is the normalized amplitude. 
The backprojections obtained from the data acquired at different transmitter positions are integrated and the final reconstructed image is obtained.

The experimentally obtained spatial distribution of the attenuation of propagating Lamb waves in the floor of the real tank is presented in Fig. 5 b. The darker areas correspond to the zones with a higher attenuation of $S_{0}$ mode Lamb waves, what is correlated to large corroded areas. The resolution of the presented image depends on the number of projections used in the tomographic reconstruction.

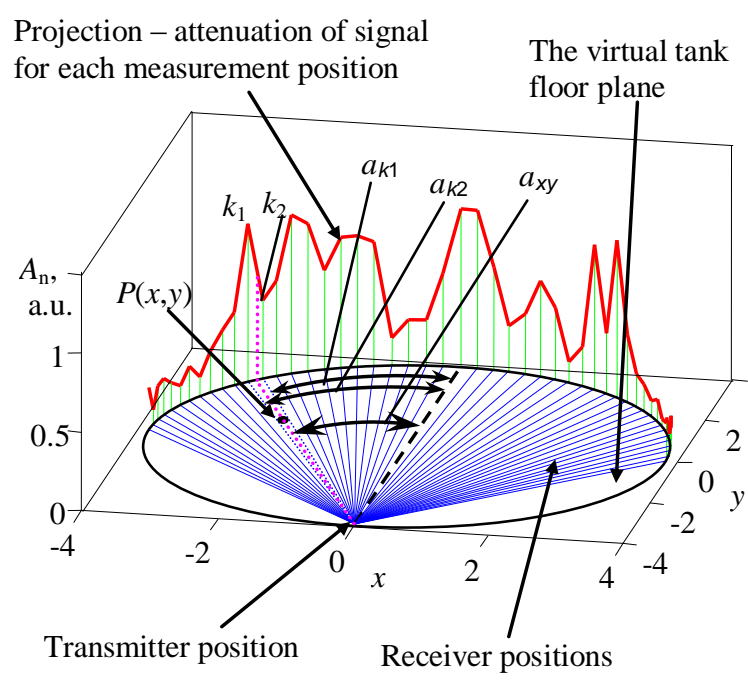

(a)
Distance, $m$

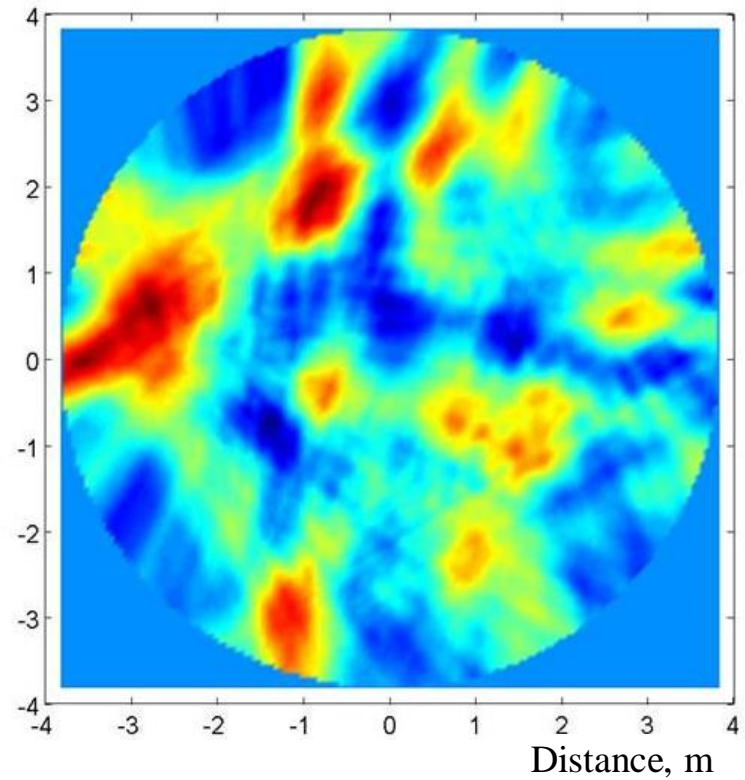

(b)

Fig.5. Spatial localisation of the tank floor non-uniformities by ultrasonic transmission tomography: a explanation of the backprojection of the single projection in the virtual tank floor plane, $\mathrm{b}$ - ultrasonic image of the petroleum tank floor reconstructed from the experimental data.

\section{Conclusions}

In order to develop an efficient NDT technique regularities of Lamb wave propagation through tank floor plates and welds loaded by sand from a bottom side and by liquid (diesel) on the top side have been investigated. The most significant signal losses occur due loading of the floor plates and due to welded seams. It has been estimated, that the attenuation function of the signal transmitted through a single weld possesses the frequency dependent harmonic oscillations due to interference of two signals: the signal transmitted through the weld and the signal reflected from the left end of the bottom plate. In order to increase accuracy of the transmission tomography, the prediction technique of the informative signal transmitted through the welded floor of the petroleum tank which takes into account attenuation, frequency dependent leakage losses and appropriate phase velocity dispersion, was proposed.

The investigation performed demonstrated that the ultrasonic tank floor inspection technique based on a tomographic reconstruction of the through transmitted Lamb waves with access only from the outside perimeter of a tank is feasible. Such technique enables to obtain the spatial distribution of zones in a welded tank floor, affected by corrosion, without emptying and cleaning the tank. It is expected that inspection of the medium size tanks, diameter of which is more than $8 \mathrm{~m}$, should be also feasible. Therefore, the necessary investigations in order to determine accuracy limits of the proposed technique and the optimal number of tomographic projections are in progress. 


\section{Acknowledgments}

The part of this work was sponsored by the European Union under the Framework-6 TANK-INSPECT and LRUCM projects. The Projects are co-ordinated and managed by TWI (UK) and are partly funded by the EC under the CRAFT programmes ref.: COOP-CT-2003-508486, COLL-CT-2005-516405.

\section{References}

[1] P. Cawley, M. J. S. Lowe, et al. Practical long range guided wave testing application to pipe and rail, Material Evaluation, Vol.61, No.1 (2003), pp. 66-74.

[2] J. Pei, M. I. Yousuf, F. L. Degertekin, B. V. Honein and B. T. Khuri-Yakub, Lamb wave tomography and its application in pipe erosion/corrosion monitoring, Research in Nondestructive Evaluation, Vol.8, No.4 (1996), pp. 189-197.

[3] R. Sicard, J. Goyette and D. Zellouf, A SAFT algorithm for Lamb wave imaging of isotropic plate-like structures, Ultrasonics, Vol. 39 (2002), pp. 487-494.

[4] R. Kažys, V. Cicènas, A. Demčenko and R. Raišutis, Attenuation of $\mathrm{S}_{0}$ Lamb wave in welded steel lap joints, Proceedings of the $28^{\text {th }}$ Scandinavian Symposium on Physical Acoustic, NTNU, Trondheim, Norway (2005), pp. 1-5.

[5] R. Kažys, L. Mažeika, R. Barauskas, R. Raišutis, V. Cicennas and A. Demčenko, 3D analysis of interaction of Lamb waves with defects in loaded steel plates, Ultrasonics, Vol.44 (2006), pp. e1127e1130.

[6] L. Mažeika, R. Kažys, R. Raišutis, A. Demčenko and R. Šliteris, Long-range ultrasonic nondestructive testing of fuel tanks, DGZfP Proceedings BB 103-CD of ECNDT 2006 (2006), pp. 1-8.

[7] E. V. Malyarenko and M. H. Hinders, Ultrasonic Lamb wave diffraction tomography, Ultrasonics, Vol. 39 (2001), pp. 269-281.

[8] R. Raišutis, R. Kažys and L. Mažeika, Application of the ultrasonic characterization methods for highly attenuating plastic materials, NDT\&International, Vol.40 (2007), pp. 324-332. 